\title{
MicroRNAs in Development
}

\author{
Danielle Maatouk and Brian D. Harfe* \\ University of Florida College of Medicine, Department of Molecular Genetics and \\ Microbiology, Gainesville, FL 32610 \\ E-mail: bharfe@mgm.ufl.edu
}

Received November 21, 2005; Revised February 27, 2006; Accepted March 2, 2006; Published March 26, 2006

\begin{abstract}
Over 10 years ago, the lab of Victor Ambros cloned an unusual gene, lin-4, which encodes two small RNA transcripts[1]. In the past few years, hundreds more of these tiny transcripts, termed microRNAs (miRNAs), have been uncovered in over a dozen species. The functions of the first two miRNAs, lin-4 and let-7, were relatively easy to identify since they were found in forward genetic screens in Caenorhabditis elegans[1,2,3]. However, uncovering the functions of the growing list of miRNAs presents a challenge to developmental biologists. This review will describe our current understanding of how miRNAs regulate gene expression and will focus on the roles these noncoding RNAs play during the development of both invertebrate and vertebrate species.
\end{abstract}

KEYWORDS: Dicer, microRNAs, mice, RISC, RNase III

\section{INTRODUCTION}

\section{MicroRNA Transcription and Processing}

MicroRNAs (miRNAs) are the newest members of the noncoding class of RNAs. The majority of miRNAs are located in intergenic regions and are transcribed from their own promoters, while a subset of miRNAs are located within the introns of protein coding genes[4,5,6]. Many of these are found in the sense orientation suggesting that they may be processed from the pre-mRNA transcript[7,8,9]. For example, in Drosophila, miR-11 is located within an intron of the E2f gene and both genes have overlapping expression patterns[7]. Similarly, miRNAs can be found in clusters that are expressed as polycistronic transcripts, with each miRNA being subsequently processed out of the primary transcript $[5,6]$. While half of the miRNAs identified in D. melanogaster are located in clusters[7], the majority of miRNAs in Caenorhabditis elegans and humans are not clustered and are located in intergenic regions[9,10].

The initial miRNA transcript forms a large hairpin structure[11,12]. This structure, termed the primary-miRNA transcript (pri-mRNA), is processed by the Microprocessor complex that is composed of the RNase III endonuclease Drosha, and the double-stranded-RNA-binding protein DGCR8 (in humans) or Pasha (in Drosophila and C. elegans)[13,14]. Within the nucleus, this complex cleaves the pri-miRNA at the base of the stem-loop, releasing the premature miRNA (pre-miRNA), a 60-70 nt stem-loop RNA molecule. The pre-miRNA is then actively transported to the cytoplasm via Exportin-5[15,16] where it is further processed by Dicer, another RNase III endonuclease[14]. Dicer cleaves the pre-miRNA approximately two helical turns from the site of Drosha cleavage, releasing the mature miRNA duplex of about $22 \mathrm{nt}[17,18,19]$. 
Once the miRNA duplex has formed, one strand is incorporated into the multiprotein RNA-Induced Silencing Complex (RISC). RISC is composed of a number of proteins, including Argonaute, and facilitates the association of the miRNA with its target genes (reviewed in [20]). While rarely found in animals, perfect complimentarity between the miRNA and its target sequence results in Argonautemediated cleavage of the target transcript between nucleotides 10-11 of the miRNA[21,22]. More commonly, imperfect pairing between the miRNA and its target results in inhibition of gene translation[2,23] and sequestration of miRNA-bound transcripts in cytoplasmic P-bodies, which are sites of mRNA degradation[24]. However, recent work by Bagga et al.[25] has clearly shown that two miRNAs (lin-4 and let-7) thought to function by inhibiting protein synthesis instead cause the degradation of their target transcripts. Interestingly, unlike the RISC-mediated endonuclease cleavage that occurs on perfect miRNA-target binding, Bagga et al. did not detect cleavage products, suggesting that target mRNA degradation may occur by a 5'-3' exonuclease-dependent mechanism. The colocalization of RISC components with cytoplasmic P-bodies supports this model[26]. Additionally, disruption of P-bodies has recently been shown to cause defects in RISC-mediated silencing[27].

\section{MICRORNAS IN DEVELOPMENT}

\section{C. elegans}

\section{Developmental Timing}

In C. elegans, miRNAs, as well as exogenous double-stranded RNAs (dsRNAs), are processed by the $C$. elegans Dicer gene, dcr-1[17,19,28]. Removal of Dicer, through direct mutation of the Dicer locus[17,28] or depletion by RNA interference (RNAi)[19] results in animals that are unable to generate an RNAi response and do not process miRNAs such as lin-4 and let-7. Mutant worms have defects in oogenesis and are sterile, suggesting a role for miRNAs and/or RNAi in germline development[17,28]. In addition, loss of dcr-1 results in heterochronic defects as a result of the misregulation of miRNA target genes[17,19,28].

The first two miRNAs to be discovered were identified as a result of their involvement in developmental timing in C. elegans. During the late L1 stage, the lin-4 miRNA is upregulated and binds to the 3' UTR (untranslated region) of the lin-14 gene, resulting in translational inhibition[2] and degradation of the lin-14 transcript[25]. Another miRNA, let-7, was similarly found to regulate developmental timing in C. elegans during the L4-to-adult transition by negatively regulating lin-14, lin28, and lin-41 through interactions with their 3' UTRs[3]. Recently, two labs have characterized a number of additional miRNAs involved in the C. elegans heterochronic pathways; mir-48, mir-84, and mir-241 were found to share a high degree of sequence similarity to let-7 at their 5 ' ends. The region of similarity includes an exact, 8-nt match in the part of the miRNA that has been postulated to be essential for target site selection[29,30]. Mutational analysis revealed that these let-7 family members have redundant functions in regulating the L2-L3 transition in C. elegans and may also function with let-7 to regulate the L4-adult transition[31,32].

The $5^{\prime}$ ends of the let-7 family members are highly conserved and may act together to repress a common target, hbl-1[32]. miRNAs bind target sequences with imperfect complementarity, however, several groups have observed regions of perfect complimentarity between the 5' end of the miRNA and the 3' UTR of the target gene[33,34,35,36,37]. Recently, Lewis et al. used a bioinformatics approach to test the hypothesis that the $5^{\prime}$ portion of the miRNA is essential for target site recognition[29,30]. Using TargetScan, a computer algorithm for predicting miRNA target genes, they found the most reliable heptamer sequence for miRNA target prediction resided at the 5' end of the miRNA (nucleotides 2-8). This observation may explain the functional redundancy reported for the let-7 family in C. elegans. Variances in their 3' sequences may allow each miRNA to carry out a different function. For example, unlike mir-48, mir-84, and mir-271, let-7 can target lin-41 through significant complimentarity to the 3' end of the let-7 miRNA[32,38]. These observations suggest that conservation at the $5^{\prime}$ end of miRNAs 
may contribute to functional redundancy between related members, while sequence variation at the 3 ' end allows for family members to target distinct genes[33,34].

\section{Neuronal Cell Fate}

A conserved feature of nervous systems is that while they develop in a morphologically symmetrical pattern, they exhibit functional asymmetry[39]. In C. elegans, the ability to respond to different chemosensory cues is controlled by two bilateral taste receptor neurons[40]. Although both neurons arise from a common precursor state, they acquire the ability to respond to different environmental signals by differential expression of guanyl cyclase receptor genes (gcy). To elucidate how asymmetry is established in the two neurons, ASE right (ASER) and ASE left (ASEL), Johnston and Hobert[41] conducted a genetic screen to identify neuronal asymmetry mutants. Identification of the lsy-6 mutant resulted in an ASEL to ASER transformation and expression of the ASER-specific gcy-5 gene in both ASE neurons. Surprisingly, cloning of the mutation revealed that lsy-6 encoded a miRNA that is normally asymmetrically expressed only in ASEL. The lsy-6 miRNA was found to have target sites in the homeobox gene $\operatorname{cog}-1$, which in turn sets off a signaling cascade resulting in the differential gcy expression patterns. Therefore, in the ASEL, lsy-6 inhibits cog- 1 and results in expression of $g c y-7$. In the ASER, there is no expression of lsy-6; therefore, $\operatorname{cog}-1$ is expressed, leading to expression of gcy-5.

Another mutant, ot26, also results in an ASEL to ASER transformation phenotype[42]. Cloning of this mutation identified the $\mathrm{C} 2 \mathrm{H} 2$ zinc-finger transcription factor die-1. die-1 was found to regulate expression of the lsy-6 miRNA, thus asymmetric die-1 expression resulted in asymmetric lsy-6 expression. Increasing the complexity of the pathway responsible for ASE asymmetry, die-1 was found to be the target of mir-273[42]. Interestingly, while mir-273 seemed to be at the top of the signaling cascade, removal of lsy- 6 resulted in symmetrical mir-273 expression, such that expression was derepressed in the ASEL due to activation of the lsy-6 target, cog-1[43]. Therefore, asymmetric gcy expression in the ASER and ASEL neurons is regulated by a complex feedback loop involving two asymmetrically expressed miRNAs (Fig. 1). This new role for miRNAs provides insight into possible mechanisms controlling the functional asymmetry of the nervous system.

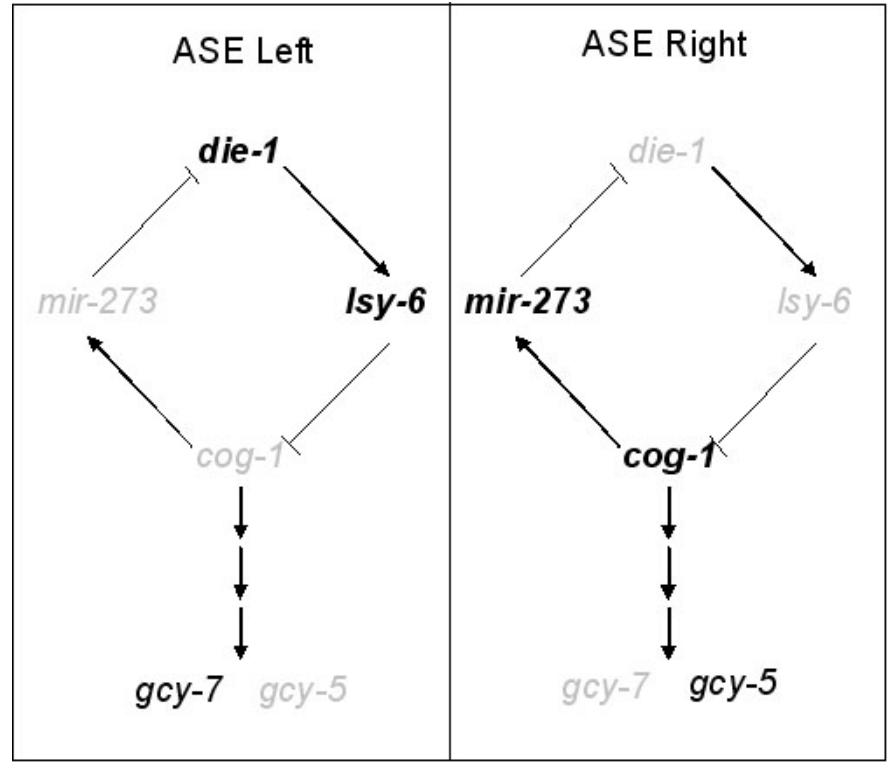

FIGURE 1. ASE neuronal cell fate in C. elegans is controlled by a feedback loop. Summary of pathway generating gcy asymmetry in the ASEL and ASER. See text for details. Genes listed in black represent expressed genes. Genes in grey are not expressed. 


\section{Drosophila}

\section{Germline Stem Cell Maintenance}

Unlike C. elegans, zebrafish, and mammals, there are two homologs of dicer in Drosophila: dicer-1 and dicer-2[44]. Dicer-2 processes small interfering RNA (siRNA) precursors such as long dsRNAs, and dicer-2 mutants are viable and fertile. Dicer-1, complexed with the dsRNA binding protein (dsRBP) Loquacious (loqs), processes endogenous miRNAs and may partially function in the siRNA pathway[45,46]. Mutations in dicer-1 cause developmental abnormalities in the eye and surrounding sensory bristles, as well as patterning defects[44]. Similar to C. elegans, deletion of dicer-1 results in germline defects. Hatfield et al.[47] observed that female dicer-1 mutants had reduced numbers of gametes, however, no defects were apparent in germline stem cell (GSC) maintenance or survival; GSC were still present in both ovaries and testes and were able to maintain their stem cell fate. Instead, the frequency of GSC cell division was reduced at the G1/S cell cycle checkpoint in dicer-1 null flies.

Transition through the G1/S checkpoint occurs by repression of the checkpoint inhibitor Dacapo (Dap)[48]. Interestingly, Dap contains multiple miRNA binding sites in its 3' UTR and a transgene lacking these sites mimics the phenotype observed on loss of dicer-1, suggesting that miRNAs may directly regulate the GSC cell cycle by repressing the checkpoint inhibitor Dap [47].

Drosophila loqs mutations result in the inability to process miRNAs[49]. The phenotype of loqs mutant flies is similar to the germline defects observed in dicer-1 null flies[45]. In the mutant, ovaries are devoid of GSCs, however, small numbers of germ cells are present within the gonad. This suggests that GSCs must have been present at an earlier stage, and loss of miRNA processing caused GSCs to either die or differentiate into another cell type[45]. Therefore, like C. elegans where loss of Dicer also results in sterility, miRNA processing plays a crucial role in the differentiation and maintenance of the Drosophila germline. It will be interesting to see if miRNAs play similar roles in other stem cell populations.

\section{Cell Proliferation and Cell Death}

Forward genetic screens have the advantage of identifying genes based on mutant phenotypes of interest. Using such screens, two labs identified Drosophila miRNAs involved in cell proliferation and apoptosis. The first group discovered the bantam mutation during a gain-of-function screen for genes involved in tissue overgrowth, suggesting a role for bantam in cell proliferation[50]. Homozygous bantam mutants were growth retarded and died during the early pupal stage, while heterozygotes were essentially normal. Using a novel computer program to identify bantam target genes, Brennecke et al. discovered hid, a known inducer of apoptosis[50]. Hid contains multiple bantam binding sites in its 3' UTR, suggesting that one function of bantam is to decrease apoptosis during embryogenesis by negatively regulating hid. It is unlikely that bantam targeting of hid alone is sufficient to cause the tissue overgrowth phenotype observed in the mutants, because other antiapoptotic factors, which block cell death, show no increase in cell proliferation when overexpressed. Therefore, the cell-proliferative and apoptotic-blocking functions of bantam may depend on additional bantam targets.

A second gene, mir-14, has also been implicated as a suppressor of cell death in Drosophila[51]. While there is no in vivo evidence, Drice, a proapoptotic gene, has been identified as a possible mir-14 target based on the presence of mir-14 binding sites in its 3' UTR. Interestingly, in addition to the observed increase in cell death in mir-14 mutants, there is an increase in fat levels, suggesting that mir-14 may regulate fat metabolism in addition to apoptosis[51].

Bantam and mir-14 represent the first two miRNAs outside of $C$. elegans to be characterized using genetic mutations, and provide examples of how individual miRNAs can mediate various processes; bantam regulates genes involved in apoptosis and cell proliferation, while mir-14 targets genes involved in apoptosis and fat metabolism. While no bantam homologs have yet been identified in mouse or 
humans, three homologs exist in C. elegans (mir-80, mir-81, mir-82)[4,6,50]; however, the genes these miRNAs target have not been identified and their potential roles in cell proliferation are unknown.

\section{Zebrafish}

\section{Brain Morphogenesis}

miRNAs are critical regulators of developmental pathways in invertebrates. High conservation of miRNAs between invertebrate and vertebrate species suggests that miRNAs may also be important for vertebrate development. Recent experiments in zebrafish and mice have shown this to be true.

In zebrafish, targeted disruption of dicer results in a loss of miRNA processing and developmental arrest around 8 days postfertilization[52]. This is after most organ systems have formed. It has been suggested that the presence of maternal Dicer masks the role miRNAs may play in earlier aspects of embryogenesis. Giraldez et al.[53] addressed this issue in zebrafish by using a germline replacement technique to generate wild type fish containing mutant germ cells (null for dicer). These chimeric fish, although lacking dicer activity in the germline, were fertile and, on intercrossing, produced embryos lacking both maternal and zygotic dicer (MZdicer). While MZdicer mutant embryos displayed normal axis formation, severe defects were observed during gastrulation, somitogenesis, and development of the heart and brain. These results suggest that, unlike C. elegans and Drosophila, Dicer is not required for germ cell development in zebrafish. Early patterning and cell specification occurred normally in MZdicer mutants, but later morphogenic processes were disrupted, suggesting that miRNAs play important roles in differentiation or maintenance of specific tissues.

The MZdicer mutant lacks all mature miRNAs. Therefore, it was not possible to characterize the roles of individual miRNAs during embryonic development. To assess what specific miRNAs might contribute to the embryonic defects observed in the MZdicer mutant, Giraldez et al.[53] used a complementation approach in which miRNAs were cloned from several early developmental stages and injected into MZdicer zebrafish in an attempt to rescue the mutant phenotypes. One family identified in this screen, the mir-430 family, was found to be highly expressed during gastrulation and somitogenesis. To determine whether loss of mir-430 processing caused the defects observed in the MZdicer mutant, mir-430 duplexes were injected into mutant embryos. Interestingly, the mir-430 duplexes rescued defects in brain development and partially rescued defects in gastrulation and somitogenesis; however, heart defects remained unchanged[53]. These results implicate the mir-430 family in controlling specific aspects of zebrafish morphogenesis. Further investigation of miRNAs and their target genes in the MZdicer mutant will help to elucidate the mechanisms by which miRNAs control morphogenic processes such as development of the zebrafish brain.

\section{Mice}

\section{Embryonic Development}

To determine the functional importance of miRNAs in a mammalian system, several labs have generated mice deficient in Dicer. Loss of Dicer results in animals that cannot process miRNAs and the misexpression of downstream miRNA target genes[54,55,56,57]. Like C. elegans and zebrafish, mice contain a single dicer gene[58]. Targeted deletion of the second RNase III domain of Dicer results in embryonic lethality prior to E7.5, with a loss of expression of the stem cell marker Oct4[54]. Maternally contributed Dicer may allow for development prior to E7.5. The absence of development past E7.5 and lack of Oct4 expression suggests that Dicer and miRNAs are key regulators of embryonic development and possibly of stem cell maintenance[54]. 
A second, independent Dicer deletion allele was recently constructed in which exons one and two were removed. Embryos homozygous for this allele produce a partially functional protein that leads to embryonic lethality between E12.5 and E14.5[57]. This mutation results in defects in blood vessel formation in yolk sacs as well as in the embryo proper. Branching vessels were observed in mutant embryos, suggesting that early stages of vessel formation were unaffected. Several genes involved in vasculogenesis and angiogenesis were misregulated in the mutants, including flt1, kdr, vegf, and tie-1. While the hypomorphic mutation results in prolonged viability, mutant embryos do not survive until birth, reinforcing the importance of miRNAs during embryonic development. Additionally, data obtained from this hypomorphic allele indicate that miRNAs play a role in the maintenance of blood vessels during embryogenesis.

\section{Limb Development}

The early embryonic lethality of Dicer mouse mutants prevented the examination of miRNA functions at later developmental stages. Our lab, in collaboration with Mike McManus (UCSF) generated a conditional allele of dicer[56]. This allele allowed us to remove Dicer from a number of locations in the mouse limb using cre recombinase (see [59] for an excellent review of site-specific recombinases). In tissues from which Dicer was removed, miRNAs were not processed. Using the Dicer conditional allele in combination with various cre alleles, we were able to analyze the effects of miRNAs in the development of various mouse tissues. To date, we have removed Dicer specifically from the limb mesoderm and ectoderm in an attempt to determine the potential role for miRNAs in limb morphogenesis and patterning.

Using a limb-mesodermal prxcre transgene, conditional mice were generated that were null for dicer in the entire limb mesoderm[56]. This resulted in a significant decrease in limb size compared to wild type littermates. This size difference can, in part, be explained by a marked increase in cell death within the mesoderm of limbs that lack Dicer. Interestingly, skeletal preparations of mutant limbs revealed that, while strikingly smaller than normal, most components of the limb were present, although there was a reduction in the number of forelimb digits. Using a conditional allele of dicer, we showed that loss of miRNA processing specifically in the limb mesoderm results in proper patterning of proximal limb components, but increased apoptosis within the mesoderm and a significant loss of forelimb digits.

Two signaling centers have been identified that control patterning of the limb bud: the zone of polarizing activity (ZPA), which controls anterior-posterior patterning and digit specification, and the apical ectodermal ridge (AER), which controls proximal-distal patterning and limb outgrowth[60]. To examine whether miRNAs in either of these signaling centers control limb patterning, Dicer was specifically removed from the ZPA or the AER[56]. Removal of Dicer from the ZPA resulted in limbs containing all five digits, however, digits four and five, which are composed of cells from the ZPA, were reduced in size due to an increase in cell death. Removal of Dicer in the AER resulted in limbs that were phenotypically indistinguishable from wild type. These results suggest that miRNAs in these signaling centers do not control digit specification or limb patterning. However, while both cre transgenes turn on at approximately E9.5, Dicer protein and/or mature miRNAs may persist following cre recombination of Dicer, depending on their rate of turnover. This is consistent with preliminary data from our lab where, using Affymetrix GeneChips, changes in gene expression were not detectable until almost 2 days after transcription of functional dicer mRNA had ceased. Use of cre alleles that express in the precursors of cells that give rise to the ZPA and AER are needed to further analyze the role miRNAs may be playing in these structures. This is especially true for cells of the AER which exist only during a limited time in early limb development. 


\section{Hematopoeitic Lineage Differentiation}

Lymphocytes mediate the immune response through the recognition of foreign antigens by receptors on their cell surface[61]. B cells and T cells are lymphocytes that arise from a common lymphoid precursor cell, but mature in different locations in the body (B cells mature in the bone marrow, while $\mathrm{T}$ cells mature in the thymus). Due to the maturation of $\mathrm{B}$ and $\mathrm{T}$ cells in different locations, different sets of receptors are expressed in these two cell populations. Generating and maintaining appropriate numbers of both cell types is essential for proper immune function. Recently, miRNAs have been implicated in influencing the differentiation of lymphoid cell types.

To study the roles of miRNAs during T cell development, Cobb et al. generated a conditional allele of dicer[55]. Using a cre transgene specifically expressed during the earliest stages of $\mathrm{T}$ cell development, they were able to assess the effects of Dicer loss on $T$ cell differentiation. In the thymus, immature $T$ cells differentiate to express one of two T cell receptors: $\alpha \beta$ or $\gamma \delta[61]$. The $\alpha \beta$-expressing cells compose over $95 \%$ or the $\mathrm{T}$ cell population and play a critical role in antigen response. The $\gamma \delta$ cell population represents approximately $5 \%$ of the $\mathrm{T}$ cell population and is present in epithelial cells, where their function is not completely understood[61]. Removal of dicer in early $\mathrm{T}$ cells results in a decreased number of $\alpha \beta$ expressing T cells, causing an increase in the percentage of $\gamma \delta$-expressing cells[55]. The reduced numbers of $\alpha \beta$ T cells may be due to increased cell death as dicer mutant thymocytes undergo increased apoptosis. Therefore, although loss of miRNA processing caused increased cell death specifically in $\alpha \beta \mathrm{T}$ cells, $\gamma \delta$ cells were unaffected. Additionally, surviving $\alpha \beta$ cells could further differentiate into cytotoxic and helper $\mathrm{T}$ lymphocytes, suggesting that miRNAs play a limited role in controlling $\mathrm{T}$ cell lineage choice.

To identify miRNAs important for hematopoeitic cell differentiation, several groups have cloned miRNAs from the bone marrow, thymus, and spleen. miRNAs were found to be expressed differentially at various stages of hematopoeitic differentiation $[8,62,63]$. One miRNA, mir-181, was upregulated as the lymphoid precursor cell differentiates into B cells[62]. To assess the function of this miRNA on B cell development, mir-181 was ectopically expressed in the hematopoietic progenitor cells. This resulted in a twofold increase of B cells with no increase in T cells. Conversely, two other miRNAs identified in their screen had the opposite result; overexpression of mir-142 or mir-223 caused a 30-40\% increase in $\mathrm{T}$ lineage cells with no effect on B lineage cells. Chen et al.[62] further tested their results in vivo. Irradiated mice were reconstituted with virally infected lymphoid progenitor cells overexpressing mir-181. In these mice, B cell numbers increased twofold over controls, while T cell numbers dropped $88 \%$. These results strongly implicate mir-181 in controlling B cell lineage choice and suggest that other miRNAs, such as mir-142 and mir-223, may also be involved in lymphoid cell differentiation.

\section{Neural Cells}

Some miRNAs are expressed in highly tissue-specific patterns, suggesting that they may function in celltype-specific differentiation. Several groups have shown that miRNAs are highly enriched in the brain and that many of these miRNAs are expressed in spatially and temporally restricted patterns[64,65,66]. For example, both neurons and astrocytes (a subtype of glial cell that supports neurons) arise from a common precursor stem cell[67]. Neurons, however, are enriched for mir-124a and mir-128, while astrocytes express mir-23, mir-66, and mir-69[67]; mir-124a is highly expressed in the brain[65,66]. Interestingly, overexpression of mir-124a in HeLa cells results in downregulation of over 170 genes[68]. The dramatic change in gene expression caused by overexpression of a single miRNA highlights how individual miRNAs may function to regulate large numbers of target genes. In HeLa cells, mir-124a overexpression led to decreased expression of genes normally expressed at very low levels in the brain. The resulting HeLa cells acquired gene expression profiles that resembled those of mature neurons. Therefore, rather than promoting differentiation, miRNAs in the brain may function to silence nonneuronal genes and help to maintain the neuronal cell state. 


\section{Insulin Secretion}

Pancreatic islet cells, made up of glucagon-secreting $\alpha$ cells and insulin-secreting $\beta$ cells, maintain the proper balance of blood glucose levels[69]. Increased uptake of glucose by $\beta$ cells leads to the secretion of insulin-containing vesicles by exocytosis. Recent data suggest that an islet-specific miRNA may contribute to insulin secretion by $\beta$ cells [70].

To explore the roles of miRNAs in regulating glucose metabolism, Poy et al.[70] cloned miRNAs from the glucose-responsive pancreatic $\beta$ cell line MIN6 and the $\alpha$ cell line TC-1. From this screen, mir375 was identified as being an islet-specific miRNA. To determine if this novel miRNA was involved in glucose metabolism, mir-375 expression was downregulated in MIN6 cells using siRNAs. Surprisingly, following glucose stimulation, MIN6 cells with decreased mir-375 had increased insulin secretion compared to control cells. Conversely, mir-375 overexpression resulted in decreased insulin secretion. Further analysis revealed that while no defect was observed in insulin synthesis or in intracellular $\mathrm{Ca}^{2+}$ signaling (essential for exocytosis of secretory vesicles[71]), there was a block in exocytosis on overexpression of mir-375. Using a computer algorithm to search for miRNA target genes that might be involved in the exocytosis defect, myotrophin (Mtpn) was identified as a mir-375 target[70]. Decreased expression of Mtpn in MIN6 cells resulted in reduced insulin secretion, mimicking mir-375 overexpression. Therefore, in MIN6 cells, mir-375 can effect insulin secretion by blocking exocytosis of insulin-containing vesicles, possibly by repressing its target gene, Mtpn. The identification of an isletspecific miRNA involved in insulin secretion adds new complexity to the $\beta$ cell pathway regulating glucose metabolism and provides additional therapeutic targets for metabolic disorders such as diabetes.

\section{Genomic Imprinting}

Mammalian development requires the proper inheritance of a maternal and paternal genome[72]. Embryos inheriting two maternal (gynogenetic) or two paternal genomes (androgenetic) die during early stages of development[72]. The nonequivalence of the maternal and paternal genomes can be attributed to the monoallelic expression of specific genes based on their "parent of origin", a phenomenon known as genomic imprinting[73]. Recently, several miRNAs were identified in a region subject to genomic imprinting, the Dlk1-Gtl2 imprinted locus[74,75]. Within this locus, the protein-coding genes Dlk1, Dio3, and Peg11 (Rtl1) are expressed exclusively from the paternal chromosome, while several noncoding RNA transcripts, Gtl2, Mirg, and anti-Peg11, are expressed maternally[76]. Seitz et al.[75] identified several miRNAs transcribed within the maternally expressed anti-Peg11 and Mirg noncoding transcripts. Interestingly, mir-136 and mir-127, encoded within the anti-Peg11 transcript, exhibit perfect complimentarity with Peg-11. Cloning and sequencing of Peg11 cleavage products corresponding to the anti-Peg11-encoded miRNAs confirmed that the imprinted miRNAs regulated Peg11 transcription by RISC-mediated cleavage of the transcript[75].

The Dlk1-Gtl2 imprinted region includes the callipyge (CLPG) locus[77]. Originally identified in sheep, the callipyge mutation leads to muscle overgrowth, possibly resulting from increased expression of the Dlk1 and Peg11 genes[78]. However, the mutation only results in a phenotype in the heterozygous state when paternally inherited. A recent publication provides a new explanation for the complex inheritance of the callipyge phenotype[74]. When the mutation is inherited maternally, increased levels of anti-Peg11-encoded miRNAs are observed. Conversely, paternal inheritance results in increased Peg11 expression, resulting in the muscle hypertrophy phenotype. When the mutation is homozygous, increased levels of miRNAs, derived from the anti-Peg11 transcript, target the overexpressed Peg11 transcript for degradation. This results in decreased Peg11 transcript, increased Peg11 cleavage products and no hypertrophic phenotype. Therefore, miRNAs in this imprinted locus act in trans to maintain the proper transcript levels of their target gene, Peg11 (Fig. 2). 


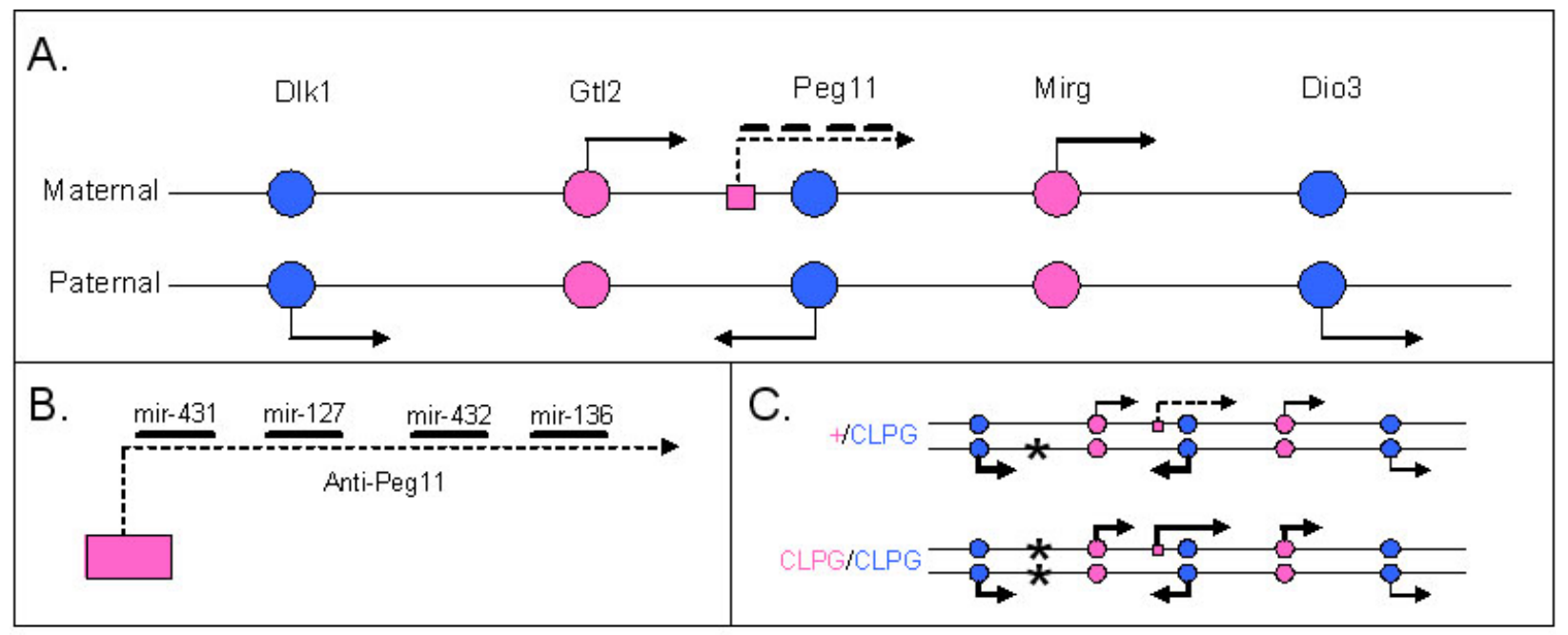

FIGURE 2. MiRNA expression in the Dlk1-Gtl2 imprinted locus. (A) Schematic representation of the maternal and paternal alleles of the Dlk1Gtl2 imprinted locus. (B) An enlarged view of the anti-Peg11 transcript including several miRNAs shown to be overexpressed when inherited with the CLPG mutation. (C) The effect of the CLPG mutation on gene expression in the Dlk1-Gtl2 locus. Only wildtype/CLPG (+/CLPG) and CLPG/CLPG are shown. See text for details. Blue circles represent paternally expressed genes. Pink circles represent maternally expressed genes. The maternally expressed anti-Peg11 transcript is depicted as a pink square and a dotted line. Genes that are transcribed are indicated with an arrow. Genes that are overexpressed are depicted by bold arrows. An asterisk $(*)$ marks the CLPG mutation.

The identification of imprinted miRNAs in the Dlk1-Peg11 locus provides an explanation for the complex inheritance of the callipyge phenotype and provides a new mechanism for a trans-acting, imprinted, noncoding RNA. As noncoding RNAs are a common theme for imprinted loci, it is possible that other imprinted, noncoding RNA transcripts may share similar regulatory roles.

\section{Muscle}

While certain miRNAs are broadly expressed, others are expressed at specific developmental stages, in a cell-type specific manner. mir-1 has been observed by several groups to be highly expressed in the heart and muscle[65,79,80]. Based on its expression pattern, mir-1 was suspected to play an important role in muscle cell differentiation, however, studies in mice and Drosophila have revealed divergent roles for this highly conserved miRNA.

In the mouse, the transcription factor Hand 2 maintains the proliferative population of undifferentiated myocytes[81,82]. Myocyte differentiation, mediated by the transcription factor Serum Response Factor (SRF) [83], is accompanied by downregulation of Hand2, which results in cessation of mitotic cell divisions[81,82]. Zhao et al.[79] recently found that the two mir-1 genes (mir-1-1 and mir-1-2) were expressed in the developing heart and that their expression was dependent on SRF binding sites in their promoters. Expression of both mir-1 genes in the somites was dependent on the transcription factors MyoD and Mef2. To understand the role of these miRNAs in cardiomyocyte development, mir-1 was overexpressed in the developing heart. Overexpression of the miRNA resulted in decreased proliferation of cardiomyocytes, heart failure, and lethality by E13.5. Using a new algorithm for identifying miRNA targets, Zhao et al. identified and validated Hand2 as a target for mir-1. Therefore, in the mouse heart, SRF activates mir-1 which then causes repression of Hand2, allowing cardiomyocytes to differentiate.

In Drosophila, mir-1 is expressed in mesodermal cells early in development, and like the mouse, the later restriction of mir-1 to heart and muscles requires the activity of mef2[80]. However, a null mutation of mir-1 had no detectable effect on heart or muscle as both tissue types were morphologically and functionally normal. Later in development, Drosophila larvae could not transition from the first instar to second instar stage, larvae became increasingly lethargic and eventually died from severe disruption of 
the body wall musculature[80]. The transition from the first to second instar stage is marked by a $\sim 200-$ fold increase in body mass over 4 days, however, the increased muscle growth is due to expansion of cell size by endocytic DNA replication (replication without cell division) and is not due to increased cell proliferation[84]. Therefore, in Drosophila, mir-1 likely is necessary for maintenance of muscle integrity or muscle cell identity. Consistent with this hypothesis, human mir-1 has been shown to restrict expression of nonmuscle genes in muscle cells[68].

The results in Drosophila seem surprising when considering the severe effect of overexpressing mouse mir-1 in the heart and with mir-1 expression being specifically expressed in the developing heart of both species. It seems that loss of mir-1 in Drosophila does not effect cardiomyocyte differentiation, or the effect is minimal, and a functional heart can still form. Although mir-1 overexpression in the mouse has significant effects on expression of its downstream target Hand2, and severe morphological defects, it will be of interest to see if loss of mir-1 also causes an early embryonic heart defect or if the phenotype will resemble that in Drosophila.

TABLE 1

miRNA Functions in C. elegans, Drosophila, Zebrafish, and Mouse

\begin{tabular}{|c|c|c|c|}
\hline Organism & miRNA(s) & miRNA Function & References \\
\hline \multirow[t]{3}{*}{ C. elegans } & $\begin{array}{l}\text { lin-4, let-7, mir-48, mir-84, mir- } \\
241\end{array}$ & Developmental timing & {$[2,3,17,19,28,31,32]$} \\
\hline & Loss of dicer & Oogenesis & {$[17,28]$} \\
\hline & Isy-6, mir-273 & Asymmetric neuronal cell fate & {$[41,42,43]$} \\
\hline \multirow[t]{3}{*}{ Drosophila } & Loss of dicer & GSC maintenance & {$[47]$} \\
\hline & bantam, mir-14 & Cell proliferation, cell death, fat metabolism & {$[50,51]$} \\
\hline & mir-1 & Muscle cell maintenance or integrity & {$[80]$} \\
\hline Zebrafish & mir-430 family & Brain morphogenesis & [53] \\
\hline \multirow[t]{7}{*}{ Mouse } & Conditional loss of dicer & Blood vessel maintenance & {$[57]$} \\
\hline & Conditional loss of dicer & Limb development & [56] \\
\hline & Conditional loss of dicer & T lymphocyte development & [55] \\
\hline & mir-181 & B lymphocyte development & {$[62]$} \\
\hline & mir-375 & Insulin secretion & [70] \\
\hline & $\begin{array}{l}\text { mir-431, mir-127, mir-432, mir- } \\
\quad 136\end{array}$ & Gene regulation in an imprinted locus & {$[74,75,85]$} \\
\hline & mir-1 & Heart and muscle development & [79] \\
\hline
\end{tabular}

\section{CONCLUSIONS}

Since the discovery and identification of hundreds of miRNAs in both invertebrate and vertebrate species, the functional importance of this new class of noncoding RNA is slowly being realized. miRNAs are now known to carry out diverse functions and even highly conserved miRNAs may play different developmental roles in different species. The analysis of organisms carrying mutations in Dicer has been a powerful approach for studying the roles of all miRNAs during development. The recent development of conditional Dicer alleles in mice will further our understanding of miRNA functions in specific tissues. However, uncovering the roles of individual miRNAs remains challenging due to possible functional redundancy between miRNAs. This problem is highlighted in the mouse model system in which it is expensive and time consuming to create multiple knockout lines. Deletion mutants will nevertheless provide valuable information on miRNA functions and help us gain a better understanding of the roles they play during development. 


\section{REFERENCES}

1. Lee, R.C., Feinbaum, R.L., and Ambros, V. (1993) The C. elegans heterochronic gene lin-4 encodes small RNAs with antisense complementarity to lin-14. Cell 75(5), 843-854.

2. Wightman, B., Ha, I., and Ruvkun, G. (1993) Posttranscriptional regulation of the heterochronic gene lin-14 by lin-4 mediates temporal pattern formation in C. elegans. Cell 75(5), 855-862.

3. Reinhart, B.J. et al. (2000) The 21-nucleotide let-7 RNA regulates developmental timing in Caenorhabditis elegans. Nature 403(6772), 901-906.

4. Lee, R.C. and Ambros, V. (2001) An extensive class of small RNAs in Caenorhabditis elegans. Science 294(5543), $862-864$.

5. Lau, N.C. et al. (2001) An abundant class of tiny RNAs with probable regulatory roles in Caenorhabditis elegans. Science 294(5543), 858-862.

6. Lagos-Quintana, M. et al. (2001) Identification of novel genes coding for small expressed RNAs. Science 294(5543), 853-858.

7. $\quad$ Aravin, A.A. et al. (2003) The small RNA profile during Drosophila melanogaster development. Dev. Cell 5(2), 337350.

8. Lagos-Quintana, M. et al. (2003) New microRNAs from mouse and human. RNA 9(2), 175-179.

9. Lim, L.P. et al. (2003) The microRNAs of Caenorhabditis elegans. Genes Dev. 17(8), 991-1008.

10. Lim, L.P. et al. (2003) Vertebrate microRNA genes. Science 299(5612), 1540.

11. Lee, Y. et al. (2002) MicroRNA maturation: stepwise processing and subcellular localization. EMBO J. 21(17), 46634670.

12. Zeng, Y. and Cullen, B.R. (2003) Sequence requirements for micro RNA processing and function in human cells. RNA 9(1), 112-123.

13. Denli, A.M. et al. (2004) Processing of primary microRNAs by the Microprocessor complex. Nature 432(7014), 231235.

14. Lee, Y. et al. (2003) The nuclear RNase III Drosha initiates microRNA processing. Nature 425(6956), $415-419$.

15. Lund, E. et al. (2004) Nuclear export of microRNA precursors. Science 303(5654), 95-98.

16. Yi, R. et al. (2003) Exportin-5 mediates the nuclear export of pre-microRNAs and short hairpin RNAs. Genes Dev. 17(24), 3011-3016.

17. Ketting, R.F. et al. (2001) Dicer functions in RNA interference and in synthesis of small RNA involved in developmental timing in C. elegans. Genes Dev. 15(20), 2654-2659.

18. Hutvagner, G. et al. (2001) A cellular function for the RNA-interference enzyme Dicer in the maturation of the let-7 small temporal RNA. Science 293(5531), 834-838.

19. Grishok, A. et al. (2001) Genes and mechanisms related to RNA interference regulate expression of the small temporal RNAs that control C. elegans developmental timing. Cell 106(1), 23-34.

20. Filipowicz, W. (2005) RNAi: the nuts and bolts of the RISC machine. Cell 122(1), 17-20.

21. Hutvagner, G. and Zamore, P.D. (2002) A microRNA in a multiple-turnover RNAi enzyme complex. Science 297(5589), 2056-2060.

22. Elbashir, S.M., Lendeckel, W., and Tuschl, T. (2001) RNA interference is mediated by 21- and 22-nucleotide RNAs. Genes Dev. 15(2), 188-200.

23. Olsen, P.H. and Ambros, V. (1999) The lin-4 regulatory RNA controls developmental timing in Caenorhabditis elegans by blocking LIN-14 protein synthesis after the initiation of translation. Dev Biol. 216(2), 671-680.

24. Pillai, R.S. et al. (2005) Inhibition of translational initiation by Let-7 MicroRNA in human cells. Science 309(5740), $1573-1576$.

25. Bagga, S. et al. (2005) Regulation by let-7 and lin-4 miRNAs results in target mRNA degradation. Cell 122(4), 553563.

26. Liu, J. et al. (2005) MicroRNA-dependent localization of targeted mRNAs to mammalian P-bodies. Nat. Cell Biol. 7(7), 719-723.

27. Jakymiw, A. et al. (2005) Disruption of GW bodies impairs mammalian RNA interference. Nat. Cell Biol. 7(12), 1167-1174.

28. Knight, S.W. and Bass, B.L. (2001) A role for the RNase III enzyme DCR-1 in RNA interference and germ line development in Caenorhabditis elegans. Science 293(5538), 2269-2271.

29. Lewis, B.P. et al. (2003) Prediction of mammalian microRNA targets. Cell 115(7), 787-798.

30. Lewis, B.P., Burge, C.B., and Bartel, D.P. (2005) Conserved seed pairing, often flanked by adenosines, indicates that thousands of human genes are microRNA targets. Cell 120(1), 15-20.

31. Li, M. et al. (2005) Regulatory mutations of mir-48, a C. elegans let-7 family MicroRNA, cause developmental timing defects. Dev. Cell 9(3), 415-422.

32. Abbott, A.L. et al. (2005) The let-7 MicroRNA family members mir-48, mir-84, and mir-241 function together to regulate developmental timing in Caenorhabditis elegans. Dev. Cell 9(3), 403-414.

33. Brennecke, J. et al. (2005) Principles of microRNA-target recognition. PLoS Biol. 3(3), e85.

34. Doench, J.G. and Sharp, P.A. (2004) Specificity of microRNA target selection in translational repression. Genes Dev. 18(5), 504-511. 
35. Kloosterman, W.P. et al. (2004) Substrate requirements for let-7 function in the developing zebrafish embryo. Nucleic Acids Res. 32(21), 6284-6291.

36. Lai, E.C. (2002) Micro RNAs are complementary to 3' UTR sequence motifs that mediate negative posttranscriptional regulation. Nat. Genet. 30(4), 363-364.

37. Stark, A. et al. (2003) Identification of Drosophila MicroRNA targets. PLoS Biol. 1(3), E60.

38. Slack, F.J. et al. (2000) The lin-41 RBCC gene acts in the C. elegans heterochronic pathway between the let-7 regulatory RNA and the LIN-29 transcription factor. Mol. Cell 5(4), 659-669.

39. Yost, H.J. (1998) Left-right development from embryos to brains. Dev. Genet. 23(3), 159-163.

40. Bargmann, C.I. and Horvitz, H.R. (1991) Chemosensory neurons with overlapping functions direct chemotaxis to multiple chemicals in C. elegans. Neuron 7(5), 729-742.

41. Johnston, R.J. and Hobert, O. (2003) A microRNA controlling left/right neuronal asymmetry in Caenorhabditis elegans. Nature 426(6968), 845-849.

42. Chang, S. et al. (2004) MicroRNAs act sequentially and asymmetrically to control chemosensory laterality in the nematode. Nature 430(7001), 785-789.

43. Johnston, R.J., Jr. et al. (2005) MicroRNAs acting in a double-negative feedback loop to control a neuronal cell fate decision. Proc. Natl. Acad. Sci. U. S. A. 102(35), 12449-12454.

44. Lee, Y.S. et al. (2004) Distinct roles for Drosophila Dicer-1 and Dicer-2 in the siRNA/miRNA silencing pathways. Cell 117(1), 69-81.

45. Forstemann, K. et al. (2005) Normal microRNA maturation and germ-line stem cell maintenance requires Loquacious, a double-stranded RNA-binding domain protein. PLoS Biol. 3(7), e236.

46. Saito, K. et al. (2005) Processing of pre-microRNAs by the Dicer-1-Loquacious complex in Drosophila cells. PLoS Biol. 3(7), e235. Hatfield, S.D. et al. (2005) Stem cell division is regulated by the microRNA pathway. Nature 435(7044), 974-978. de Nooij, J.C., Letendre, M.A., and Hariharan, I.K. (1996) A cyclin-dependent kinase inhibitor, Dacapo, is necessary for timely exit from the cell cycle during Drosophila embryogenesis. Cell 87(7), 1237-1247.

49. Scherz, P.J. et al. (2004) The limb bud Shh-Fgf feedback loop is terminated by expansion of former ZPA cells. Science 305(5682), 396-399.

50. Brennecke, J. et al. (2003) bantam encodes a developmentally regulated microRNA that controls cell proliferation and regulates the proapoptotic gene hid in Drosophila. Cell 113(1), 25-36.

51. Xu, P. et al. (2003) The Drosophila microRNA Mir-14 suppresses cell death and is required for normal fat metabolism. Curr. Biol. 13(9), 790-795.

52. Wienholds, E. et al. (2003) The microRNA-producing enzyme Dicer1 is essential for zebrafish development. Nat. Genet. 35(3), 217-218.

53. Giraldez, A.J. et al. (2005) MicroRNAs regulate brain morphogenesis in zebrafish. Science 308(5723), $833-838$.

54. Bernstein, E. et al. (2003) Dicer is essential for mouse development. Nat. Genet. 35(3), 215-217.

55. Cobb, B.S. et al. (2005) T cell lineage choice and differentiation in the absence of the RNase III enzyme Dicer. $J$. Exp. Med. 201(9), 1367-1373.

56. Harfe, B.D. et al. (2005) The RNaseIII enzyme Dicer is required for morphogenesis but not patterning of the vertebrate limb. Proc. Natl. Acad. Sci. U. S. A. 102(31), 10898-10903.

57. Yang, W.J. et al. (2005) Dicer is required for embryonic angiogenesis during mouse development. J. Biol. Chem. 280(10), 9330-9335.

58. Nicholson, R.H. and Nicholson, A.W. (2002) Molecular characterization of a mouse cDNA encoding Dicer, a ribonuclease III ortholog involved in RNA interference. Mamm. Genome 13(2), 67-73.

59. Branda, C.S. and Dymecki, S.M. (2004) Talking about a revolution: the impact of site-specific recombinases on genetic analyses in mice. Dev. Cell 6(1), 7-28.

60. Tickle, C. (2003) Patterning systems - from one end of the limb to the other. Dev. Cell 4(4), 449-458.

61. Lewin, B. (1997) Genes VI. Oxford University Press, New York.

62. Chen, C.Z. et al. (2004) MicroRNAs modulate hematopoietic lineage differentiation. Science 303(5654), 83-86.

63. Monticelli, S. et al. (2005) MicroRNA profiling of the murine hematopoietic system. Genome Biol. 6(8), R71.

64. Krichevsky, A.M. et al. (2003) A microRNA array reveals extensive regulation of microRNAs during brain development. RNA 9(10), 1274-1281.

65. Lagos-Quintana, M. et al. (2002) Identification of tissue-specific microRNAs from mouse. Curr. Biol. 12(9), 735739.

66. Miska, E.A. et al. (2004) Microarray analysis of microRNA expression in the developing mammalian brain. Genome Biol. 5(9), R68.

67. Smirnova, L. et al. (2005) Regulation of miRNA expression during neural cell specification. Eur. J. Neurosci. 21(6), 1469-1477.

68. Lim, L.P. et al. (2005) Microarray analysis shows that some microRNAs downregulate large numbers of target mRNAs. Nature 433(7027), 769-773.

69. Bouwens, L. and Rooman, I. (2005) Regulation of pancreatic beta-cell mass. Physiol. Rev. 85(4), $1255-1270$.

70. Poy, M.N. et al. (2004) A pancreatic islet-specific microRNA regulates insulin secretion. Nature 432(7014), $226-230$.

71. Rutter, G.A. (2004) Visualising insulin secretion. The Minkowski Lecture 2004. Diabetologia 47(11), $1861-1872$. 
72. McGrath, J. and Solter, D. (1984) Completion of mouse embryogenesis requires both the maternal and paternal genomes. Cell 37(1), 179-183.

73. Cattanach, B.M. (1986) Parental origin effects in mice. J. Embryol. Exp. Morphol. 97(Suppl), 137-150.

74. Davis, E. et al. (2005) RNAi-mediated allelic trans-interaction at the imprinted Rtl1/Peg11 locus. Curr. Biol. 15(8), 743-749.

75. Seitz, H. et al. (2003) Imprinted microRNA genes transcribed antisense to a reciprocally imprinted retrotransposonlike gene. Nat. Genet. 34(3), 261-262.

76. Georges, M., Charlier, C., and Cockett, N. (2003) The callipyge locus: evidence for the trans interaction of reciprocally imprinted genes. Trends Genet. 19(5), 248-252.

77. Cockett, N.E. et al. (1996) Polar overdominance at the ovine callipyge locus. Science 273(5272), $236-238$.

78. Charlier, C. et al. (2001) The callipyge mutation enhances the expression of coregulated imprinted genes in cis without affecting their imprinting status. Nat. Genet. 27(4), 367-369.

79. Zhao, Y., Samal, E., and Srivastava, D. (2005) Serum response factor regulates a muscle-specific microRNA that targets Hand 2 during cardiogenesis. Nature 436(7048), 214-220.

80. Sokol, N.S. and Ambros, V. (2005) Mesodermally expressed Drosophila microRNA-1 is regulated by Twist and is required in muscles during larval growth. Genes Dev. 19(19), 2343-2354.

81. Srivastava, D. et al. (1997) Regulation of cardiac mesodermal and neural crest development by the bHLH transcription factor, dHAND. Nat. Genet. 16(2), 154-160.

82. McFadden, D.G. et al. (2005) The Hand1 and Hand2 transcription factors regulate expansion of the embryonic cardiac ventricles in a gene dosage-dependent manner. Development 132(1), 189-201.

83. Wang, D. et al. (2001) Activation of cardiac gene expression by myocardin, a transcriptional cofactor for serum response factor. Cell 105(7), 851-862.

84. $\quad$ Edgar, B.A. and Orr-Weaver, T.L. (2001) Endoreplication cell cycles: more for less. Cell 105(3), $297-306$.

85. Seitz, H. et al. (2004) A large imprinted microRNA gene cluster at the mouse Dlk1-Gt12 domain. Genome Res. 14(9), 1741-1748.

\section{This article should be cited as follows:}

Maatouk, D. and Harfe, B.D. (2006) MicroRNAs in development. TSW Development \& Embryology 1, 14-26. DOI 10.1100/tswde.2006.64. 

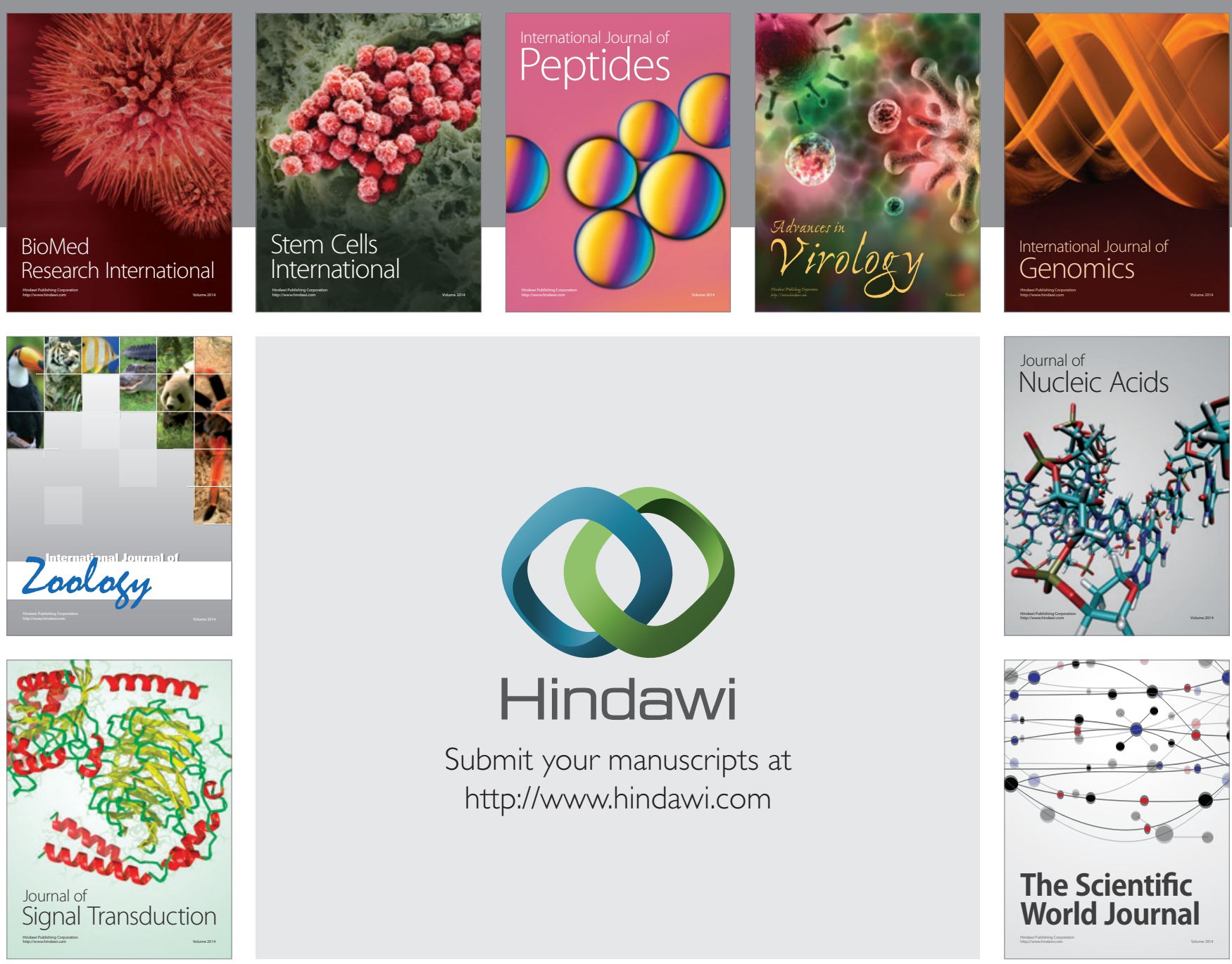

Submit your manuscripts at

http://www.hindawi.com
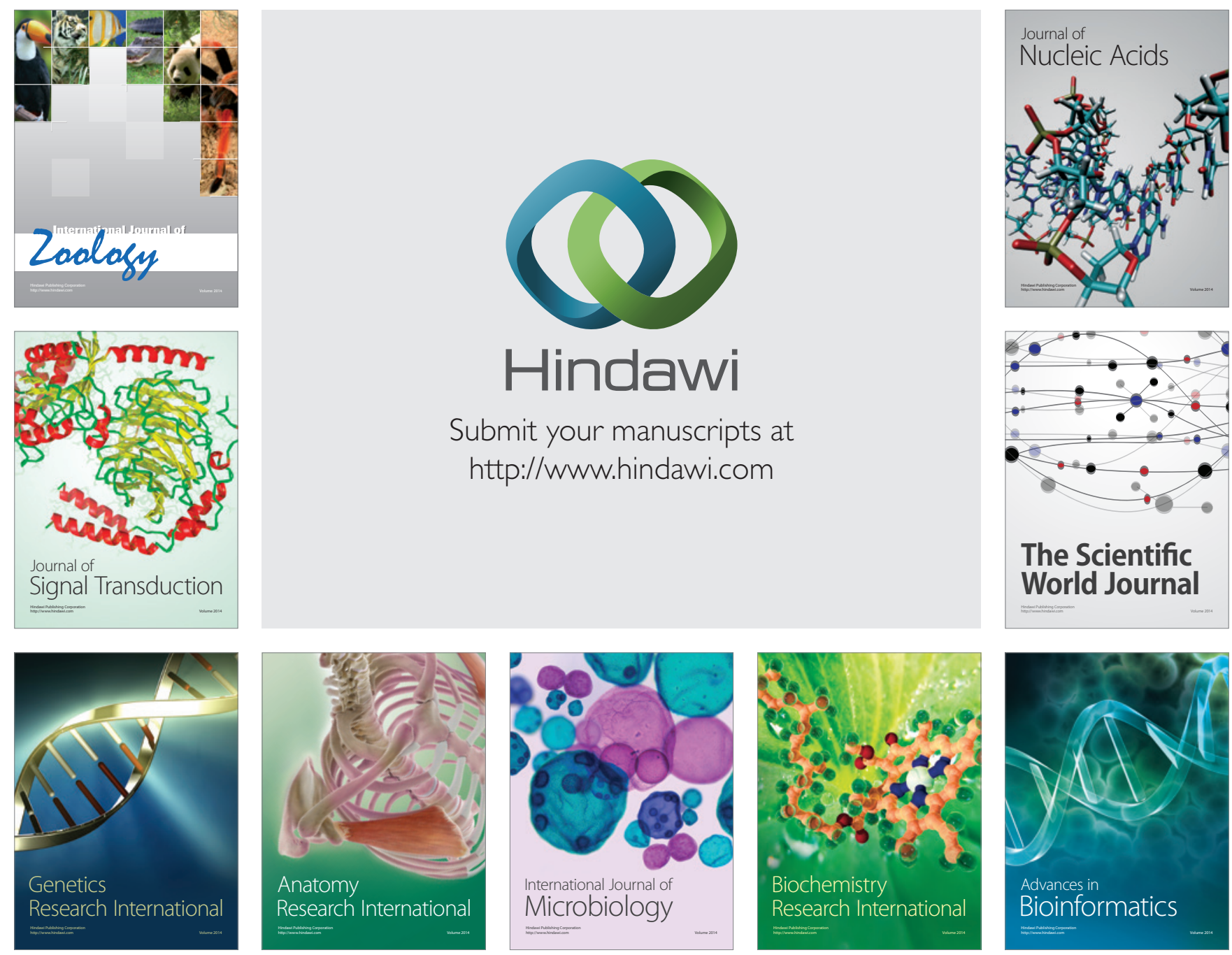

The Scientific World Journal
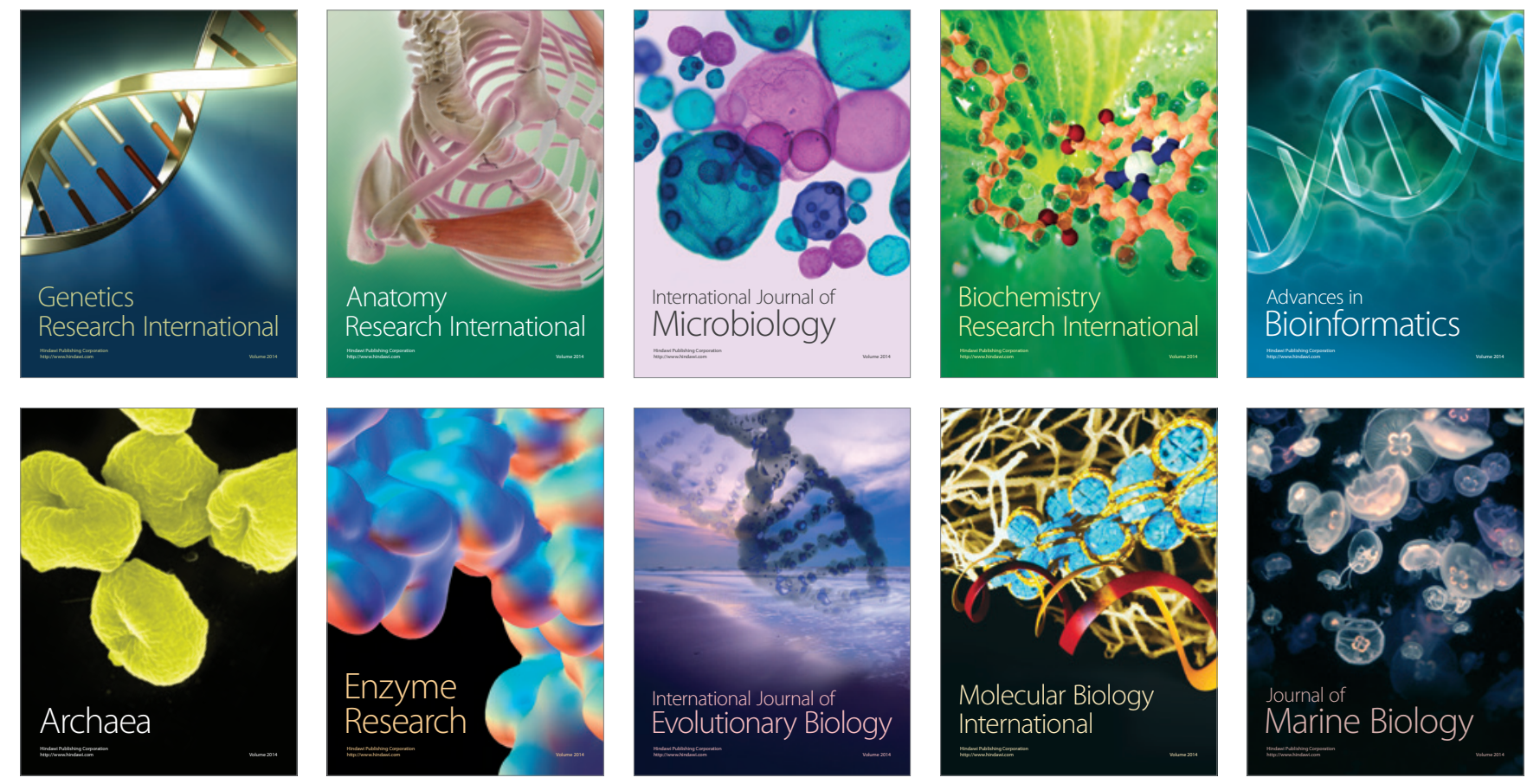